Pacific Journal of Mathematics

SOME REMARKS ON SPECIAL DISCONJUGACY CRITERIA
FOR DIFFERENTIAL SYSTEMS 


\title{
SOME REMARKS ON SPECIAL DISCONJUGACY CRITERIA FOR DIFFERENTIAL SYSTEMS
}

\author{
WILLIAM T. REID
}

The present note is devoted to the discussion of a special class of transformations that preserves the oscillatory or disconjugacy nature of solutions for a type of first order differential system in a $2 n$-dimensional vector function, and which includes as a particular instance a well-known transformation of a real scalar second order homogeneous ordinary differential equation to the canonical form $y^{\prime \prime}(t)+r(t) y(t)=0$.

It is well-known, (see, for example, Reid [4, 6, 7]), that for self-adjoint differential systems conditions of oscillation may be characterized by variational criteria, and, in particular, the property of disconjugacy is equivalent to the positive definiteness of an associated hermitian integral functional on a suitable class of vector functions. Moreover, (see, for example, Reid [4; §5]; Hartman and Wintner [2]), results for self-adjoint differential systems may be applied to yield sufficient conditions for disconjugacy in the case of nonself-adjoint systems. These criteria and procedures are of basic significance for the utilization of the considered transformations in the study of oscillation and comparison phenomena.

Matrix notation is used throughout; in particular, matrices of one column are called vectors, and for a vector $u=\left(u_{\alpha}\right),(\alpha=1, \cdots, n)$, the norm $|u|$ is given by $\left(\left|u_{1}\right|^{2}+\cdots+\left|u_{n}\right|^{2}\right)^{1 / 2}$; the linear vector space of ordered $n$-tuples of complex numbers, with complex scalars, is denoted by $\mathbf{C}_{n}$. The $n \times n$ identity matrix is denoted by $E_{n}$, or by merely $E$ when there is no ambiguity, while 0 is used indiscriminately for the zero matrix of any dimensions; the conjugate transpose of a matrix $M$ is denoted by $M^{*}$. If $M$ is an $n \times n$ matrix the symbol $\nu[M]$ is used for the maximum of $|M y|$ on the unit ball $\{y:|y| \leqq 1\}$ in $\mathbf{C}_{n}$. The notation $M \geqq N,\{M>N\}$, is used to signify that $M$ and $N$ are hermitian matrices of the same dimensions, and $M-N$ is a nonnegative, \{positive\} definite hermitian matrix. In general, if $M$ is an $n \times n$ matrix, let $\Re e$ and $\mathfrak{F} M$ denote the hermitian matrices Re $M=\frac{1}{2}\left(M+M^{*}\right)$, $\mathfrak{m} M=i / 2\left(M^{*}-M\right)$ so that $M=\Re e M+i \mathfrak{m} M$. If the elements of a matrix function $M(t)$ are a.c. (absolutely continuous) on arbitrary compact subintervals of a given interval $I$, then $M(t)$ is said to be locally a.c. on $I$; moreover, $M^{\prime}(t)$ signifies the matrix of derivatives at values where these derivatives exist and the zero matrix elsewhere. Correspondingly, if the elements of $M(t)$ are 
(Lebesgue) integrable on a compact interval $[a, b]$, then $\int_{a}^{b} M(t) d t$ denotes the matrix of integrals of respective elements of $M(t)$. If $M(t)$ and $N(t)$ are equal a.e. (almost everywhere) on their domain of definition we write simply $M(t)=N(t)$. A matrix function is called continuous, integrable, etc., when each of its elements possesses the specified property.

For a given compact interval $[a, b]$ on the real line the symbols $\mathfrak{B}_{n r}[a, b], \mathfrak{B}_{n r}^{\infty}[a, b]$ are used to denote the classes of $n \times r$ matrix functions $M(t)=\left[M_{\alpha \beta}(t)\right],(\alpha=1, \cdots, n ; \beta=1, \cdots, r)$, which on $[a, b]$ are respectively integrable, measurable and essentially bounded, where in each case measure and integral is understood to be in the sense of Lebesgue. For brevity, $\mathfrak{\&}_{n}[a, b]$ and $\mathfrak{L}_{n}^{\infty}[a, b]$ are written for $\mathfrak{\&}_{n 1}[a, b]$ and $\mathfrak{R}_{n 1}^{\infty}[a, b]$, respectively.

2. Transformations for differential systems. We shall be concerned with a vector differential system of the form

$$
\begin{aligned}
& L_{1}[u, v](t) \equiv-v^{\prime}(t)+C(t) u(t)-D(t) v(t)=0, \\
& L_{2}[u, v](t) \equiv u^{\prime}(t)-A(t) u(t)-B(t) v(t)=0,
\end{aligned}
$$

in $n$-dimensional vector functions

$$
u(t) \equiv\left(u_{i}(t)\right), v(t) \equiv\left(v_{i}(t)\right),
$$

$(i=1, \cdots, n)$. In terms of the $2 n$-dimensional vector function $y(t)=$ $\left(y_{\sigma}(t)\right),(\sigma=1, \cdots, 2 n)$, with $y_{i}(t)=u_{i}(t), y_{n+i}(t)=v_{i}(t),(i=1, \cdots, n)$, this system may be written also as

$$
\mathscr{L}[y] \equiv \mathscr{J} y^{\prime}(t)+\mathscr{A}(t) y(t)=0,
$$

where

$$
\mathscr{J}=\left[\begin{array}{cc}
0 & -E_{n} \\
E_{n} & 0
\end{array}\right], \mathscr{A}(t)=\left[\begin{array}{ll}
C(t) & -D(t) \\
-A(t) & -B(t)
\end{array}\right]
$$

Throughout the discussion it will be assumed that the coefficient matrix functions satisfy the following hypothesis.

$\left(\mathbf{H}_{1}\right) \quad A(t), B(t), C(t), D(t)$ are $n \times n$ matrix functions defined on a given interval $I$ on the real line, and are of class $\mathfrak{B}_{n n}[a, b]$ on arbitrary compact subintervals $[a, b]$ of $I$.

Corresponding to the vector differential system (2.1), one has the matrix differential system

$$
\begin{aligned}
& L_{1}[U, V](t) \equiv-V^{\prime}(t)+C(t) U(t)-D(t) V(t)=0, \\
& L_{2}[U, V](t) \equiv U^{\prime}(t)-A(t) U(t)-B(t) V(t)=0,
\end{aligned}
$$


in $n \times r$ matrix functions $U(t), V(t)$, while the associated $2 n \times r$ matrix function $Y(t)=(U(t) ; V(t))$ with

$$
Y_{i \beta}(t)=U_{i \beta}(t), Y_{n+i, \beta}(t)=V_{i \beta}(t),
$$

$(i=1, \cdots, n ; \beta=1, \cdots, r)$, is a solution of the equation

$$
\mathscr{L}[Y](t) \equiv \mathscr{J} Y^{\prime}(t)+\mathscr{A}(t) Y(t)=0 .
$$

In particular, the linear homogeneous differential system (2.1) is self-adjoint, or Hamiltonian, if the following conditions hold.

$\left(\mathbf{H}_{2}\right) \quad B(t)$ and $C(t)$ are hermitian, and $D(t)=A^{*}(t)$ for $t \in I$.

In general, two distinct points $t_{1}$ and $t_{2}$ on $I$ are said to be (mutually) conjugate with respect to (2.1) if there exists a solution $(u(t)$; $v(t))$ of this system with $u\left(t_{1}\right)=0=u\left(t_{2}\right)$, while $u(t) \not \equiv 0$ for $t$ on the subinterval with endpoints $t_{1}$ and $t_{2}$. The system (2.1) is said to be disconjugate on a subinterval $I_{0}$ provided no two distinct points of this interval are conjugate.

In the case of a self-adjoint system (2.1), one has the important fact that if $y_{\alpha}(t)=\left(u_{\alpha}(t) ; v_{\alpha}(t)\right), \alpha=1,2$, are solutions of (2.1), then the function $y_{2}^{*}(t) \mathscr{J} y_{1}(t)=v_{2}^{*}(t) u_{1}(t)-u_{2}^{*}(t) v_{1}(t)$ is constant on $I$. If the value of this constant is zero, then $y_{1}(t)$ and $y_{2}(t)$ are said to be (mutually) conjoined solutions of (2.1). In particular, if

$$
Y(t)=(U(t) ; V(t))
$$

is a $2 n \times n$ matrix which is a solution of $\left(2.1_{M}^{\prime}\right)$, and whose $n$ column vectors are linearly independent solutions of $\left(2.1^{\prime}\right)$ which are mutually conjoined, then for brevity $Y(t)$ is called a conjoined basis for (2.1). Moreover, if $Y(t)=(U(t) ; V(t))$ is a conjoined basis for (2.1), and at a point $t_{0}$ of $I$ the $n \times n$ matrix $U(t)$ is of rank $n-k$, then in accordance with the terminology of Morse [3] the point $t_{0}$ is said to be a focal point of $Y(t)$ of order $k$.

For a compact subinterval $[a, b]$ of $I$, let $\mathscr{D}_{0}[a, b]$ denote the class of $n$-dimensional vector functions $\eta(t)$ which are a.c. on $[a, b]$ and satisfy the differential equation

$$
L_{2}[\eta, \zeta](t) \equiv \eta^{\prime}(t)-A(t) \eta(t)-B(t) \zeta(t)=0
$$

with $\zeta(t) \in \mathfrak{R}_{n}^{\infty}[a, b]$, while $\eta(a)=0=\eta(b)$. Moreover, let $\mathbf{H}_{+}[a, b]$ de'note the condition that the hermitian functional

$$
J[\eta ; a, b]=\int_{a}^{b}\left\{\zeta^{*}(t) B(t) \zeta(t)+\eta *(t) C(t) \eta(t)\right\} d t
$$

is positive definite on $\mathscr{D}_{0}[a, b]$. It is to be remarked that the value of the integral in (2.3) is a function of $\eta$ on $\mathscr{D}_{0}[a, b]$, since if $\zeta_{1}(t)$ 
and $\zeta_{2}(t)$ are vector functions in $\mathfrak{R}_{n}^{\infty}[a, b]$ such that $L_{2}\left[\eta, \zeta_{\alpha}\right](t)=0$ for $\alpha=1,2$, then $\zeta_{1}^{*}(t) B(t) \zeta_{1}(t)=\zeta_{2}^{*}(t) B(t) \zeta_{2}(t)$. As is well-known, (see, for example, Reid [4; Th. 2.1]; Reid [7; Th. 5.1]), for self-adjoint differential systems (2.1) the condition of disconjugacy is characterized by certain variational conditions, that may be phrased as follows.

THEOREM 2.1. If conditions $\left(\mathbf{H}_{1}\right)$ and $\left(\mathbf{H}_{2}\right)$ hold, then for $[a, b] a$ compact subinterval of I condition $\mathbf{H}_{+}[a, b]$ holds if and only if $B(t) \geqq$ 0 for $t$ a.e. on $[a, b]$, and one of the following conditions holds:

(i) (2.1) is disconjugate on $[a, b]$;

(ii) there exists a conjoined basis $Y(t)=(U(t) ; V(t))$ for (2.1) with $U(t)$ nonsingular on $[a, b]$.

In particular, if $B(t) \geqq 0$ for $t$ a.e. on $[a, b]$, then the conditions (i), (ii) and $\mathbf{H}_{+}[a, b]$ are equivalent.

If $T(t)$ and $S(t)$ are nonsingular $n \times n$ matrix functions which are locally a.c. on $I$, then under the transformation

$$
u(t)=T(t) u^{0}(t), v(t)=S(t) v^{0}(t),
$$

we have the identities

$$
L_{1}[u, v](t) \equiv S(t) L_{1}^{0}\left[u^{0}, v^{0}\right](t), L_{2}[u, v](t)=T(t) L_{2}^{0}\left[u^{0}, v^{0}\right](t),
$$

where

$$
\begin{aligned}
& L_{1}^{0}\left[u^{0}, v^{0}\right](t) \equiv-v^{0^{\prime}}(t)+C^{0}(t) u^{0}(t)-D^{0}(t) v^{0}(t), \\
& L_{2}^{0}\left[u^{0}, v^{0}\right](t) \equiv u^{0^{\prime}}(t)-A^{0}(t) u^{0}(t)-B^{0}(t) v^{0}(t),
\end{aligned}
$$

and the coefficient matrices of $\left(2.1^{\circ}\right)$ are given by

$$
\begin{aligned}
& A^{0}=T^{-1}\left[A T-T^{\prime}\right], B^{0}=T^{-1} B S, \\
& C^{0}=S^{-1} C T, D^{0}=S^{-1}\left[S^{\prime}+D S\right] .
\end{aligned}
$$

Clearly one has the following result.

LEMMA 2.1. If $\left(\mathbf{H}_{1}\right)$ is satisfied, then distinct values $t_{1}, t_{2}$ on $I$ are conjugate with respect to (2.1) if and only if they are conjugate with respect to $\left(2.1^{\circ}\right)$; in particular, (2.1) is disconjugate on a subinterval $I_{0}$ of $I$ if and only if $\left(2.1^{\circ}\right)$ is disconjugate on this subinterval.

We shall be concerned with systems (2.1) in which the following additional condition holds.

$\left(\mathbf{H}_{3}\right)$ On $I$ the matrix function $B(t)$ is nonsingular, locally a.c., and possesses a square root matrix $B^{1 / 2}(t)$ which is locally a.c. on this interval.

It is to be remarked that if $B(t)$ is nonsingular and locally a.c. 
on $I$, while there exists a nonzero locally a.c. scalar function $\rho(t)$ such that $\nu[E-\rho(t) B(t)]<1$ for $t \in I$, then the binomial series may be used to provide a locally a.c. square root $B^{1 / 2}(t)$ of $B(t)$ with series expansion

$$
B^{1 / 2}(t)=\left[1 / \rho^{1 / 2}(t)\right]\left\{E-\sum_{k=1}^{\infty} c_{k}[E-\rho(t) B(t)]^{k}\right\},
$$

where $c_{1}=1 / 2, \quad c_{k}=[1 \cdot 3 \cdots(2 k-3)] /\left[k ! 2^{k}\right], \quad(k=1,2, \cdots),($ for example, see Reid [5; $\S 4]$ ). In particular, this condition holds whenever $B(t)$ is a positive definite hermitian matrix that is locally a.c. on $I$; in this case the corresponding square root matrix provided by this binomial series expansion is the unique positive definite hermitian square root of $B(t)$.

3. Special transformations which preserve disconjugacy. The particular type of transformation to be used in the results of this section is introduced in the following theorem.

THEOREM 3.1. If hypotheses $\left(\mathbf{H}_{1}\right)$ and $\left(\mathbf{H}_{3}\right)$ hold for (2.1), and $B^{1 / 2}(t)$ is a square root of $B(t)$ as in $\left(\mathbf{H}_{3}\right)$, with inverse $B^{-1 / 2}(t)$, then under the transformation

$$
u(t)=B^{1 / 2}(t) u^{0}(t), v(t)=B^{-1 / 2}(t) v^{0}(t),
$$

the system (2.1) is transformed into the system

$$
\begin{aligned}
& L_{1}^{0}\left[u^{0}, v^{0}\right](t) \equiv-v^{0^{\prime}}(t)+C^{0}(t) u^{0}(t)-D^{0}(t) v^{0}(t)=0, \\
& L_{2}^{0}\left[u^{0}, v^{0}\right](t) \equiv u^{0^{\prime}}(t)-A^{0}(t) u^{0}(t)-v^{0}(t)=0,
\end{aligned}
$$

where

$$
\begin{aligned}
& A^{0}=B^{-1 / 2}\left\{A B^{1 / 2}-\left[B^{1 / 2}\right]^{\prime}\right\}, C^{0}=B^{1 / 2} C B^{1 / 2}, \\
& D^{0}=\left[-\left(B^{1 / 2}\right)^{\prime}+B^{1 / 2} D\right] B^{-1 / 2} .
\end{aligned}
$$

Now let the matrix functions $A_{1}(t)$ and $A_{2}(t)$ be defined as

$$
\begin{aligned}
& A_{1}(t)=\frac{1}{2}\left[A^{0}(t)+A^{0 *}(t)\right]=\Re e\left(A^{0}(t),\right. \\
& \mathrm{A}_{2}(t)=\frac{1}{2}\left[A^{0}(t)-A^{0 *}(t)\right]=i \mathfrak{s} A^{0}(t),
\end{aligned}
$$

so that $A^{0}(t)=A_{1}(t)+A_{2}(t)$ and $A^{0 *}(t)=A_{1}(t)-A_{2}(t)$. We shall also assume that we have satisfied the further differentiability condition $\left(\mathbf{H}_{4}\right) \quad A_{1}(t)=\Re e A^{0}(t)$ is locally a.c. on $I$.

In particular, $\left(\mathbf{H}_{4}\right)$ holds when $A^{\circ}(t)$ is locally a.c. on $I$, and when in addition to the previous condition on $B(t)$ we have that $\left[B^{1 / 2}(t)\right]^{\prime}$ is locally a.c. on $I$. In turn, this latter condition holds when $B(t)$ and the associated nonzero scalar function $\rho(t)$ appearing in the discussion 
following $\left(\mathbf{H}_{3}\right)$ are individually continuously differentiable on $I$, and the respective derivative matrix and scalar function are locally a.c. on $I$.

The following result may be verified directly.

Lemma 3.1. If hypotheses $\left(\mathbf{H}_{1}\right),\left(\mathbf{H}_{3}\right)$ and $\left(\mathbf{H}_{4}\right)$ hold for the differential system (2.1), then under the transformation

$$
u^{0}(t)=u_{1}(t), v^{0}(t)=v_{1}(t)-A_{1}(t) u_{1}(t),
$$

the system (3.2) becomes

$$
\begin{aligned}
-v_{1}^{\prime}(t)+C_{1}(t) u_{1}(t)-D_{1}(t) v_{1}(t) & =0, \\
u_{1}^{\prime}(t)-A_{2}(t) u_{1}(t)-v_{1}(t) & =0,
\end{aligned}
$$

where

$$
\begin{aligned}
& C_{1}(t)=C^{0}(t)+A_{1}(t) A_{2}(t)+D^{0}(t) A_{1}(t)+A_{1}^{\prime}(t), \\
& D_{1}(t)=D^{0}(t)-A_{1}(t) .
\end{aligned}
$$

Now let $M(t)$ be the solution of the first order differential system

$$
M^{\prime}(t)-A_{2}(t) M(t)=0, M\left(t_{0}\right)=E,
$$

where $t_{0}$ is some fixed point of $I$. Since $A_{2}^{*}(t)=-A_{2}(t)$, the solution $M(t)$ of (3.8) is unitary for $t \in I$, so that

$$
M(t) M^{*}(t) \equiv E \equiv M^{*}(t) M(t) \text {. }
$$

Moreover, under the transformation

$$
u_{1}(t)=M(t) u_{2}(t), v_{1}(t)=M(t) v_{2}(t),
$$

the system (3.6) becomes

$$
\begin{aligned}
-v_{2}^{\prime}(t)+C_{2}(t) u_{2}(t)-D_{2}(t) v_{2}(t) & =0, \\
u_{2}^{\prime}(t)-v_{2}(t) & =0,
\end{aligned}
$$

where the matrix functions $C_{2}(t)$ and $D_{2}(t)$ are given by

$$
C_{2}=M^{*} C_{1} M, D_{2}=M *\left[A_{2}+D_{1}\right] M=M^{*}\left[D^{0}-A^{0 *}\right] M .
$$

In particular, $\left(u_{2}(t) ; v_{2}(t)\right)$ is a solution of $(3.10)$ if and only if $x(t)=$ $u_{2}(t)$ is a solution of the linear homogeneous second order vector differential equation

$$
x^{\prime \prime}(t)+D_{2}(t) x^{\prime}(t)-C_{2}(t) x(t)=0,
$$

and $v_{2}(t)=x^{\prime}(t)$.

Combining these results, we have the following theorem. 
THEOREM 3.2. If hypotheses $\left(\mathbf{H}_{1}\right),\left(\mathbf{H}_{3}\right)$ and $\left(\mathbf{H}_{4}\right)$ hold, and $M(t)$ is the solution of the differential system (3.8), then $(u(t)$; $v(t))$ is a solution of (2.1) if and only if

$$
x(t)=M^{*}(t) B^{-1 / 2}(t) u(t)
$$

is a solution of (3.12), with $x^{\prime}(t)=M^{*}(t)\left[B^{1 / 2} v(t)+A_{1}(t) M(t) x(t)\right]$.

From the form of the transformations occurring in (3.1), (3.5) and (3.9), it follows readily that distinct points $t_{1}, t_{2}$ on $I$ are conjugate with respect to (2.1) if and only if these points are conjugate with respect to the system (3.6) and (3.9). Also, (2.1) is disconjugate on a subinterval $I_{0}$ if and only if the systems (3.6) and (3.9) are disconjugate on $I_{0}$. In particular, from $\S 5$ of Reid [4] we have the result of the following theorem. For systems (2.1) with real coefficient matrices, the conclusion of this theorem with $\lambda=0$ is also a consequence of results of Hartman and Wintner [2].

THEOREM 3.3. Suppose that hypotheses $\left(\mathbf{H}_{1}\right),\left(\mathbf{H}_{3}\right)$ and $\left(\mathbf{H}_{4}\right)$ hold. Then (2.1) is disconjugate on $I$ if for each compact subinterval $[a, b]$ of $I$ there exists a corresponding real constant $\lambda$ such that the selfadjoint equation

$$
\left[x^{\prime}(t)+Q(t, \lambda) x(t)\right]^{\prime}-\left[Q^{*}(t, \lambda) x^{\prime}(t)+P(t, \lambda) x(t)\right]=0
$$

is disconjugate on this interval, where

$$
\begin{aligned}
& P^{\prime}(t, \lambda)=\Re \mathrm{e} C_{2}(t)+\lambda \Im \mathfrak{m} C_{2}(t), \\
& Q(t, \lambda)=-\frac{1}{2}(1+\lambda i) D_{2}^{*}(t) .
\end{aligned}
$$

4. Self-adjoint differential systems. If in the original differential system (2.1) we have $D(t) \equiv A^{*}(t)$, then whenever hypotheses $\left(\mathbf{H}_{1}\right)$ holds and $B(t)$ is positive definite and locally a.c. we have

$$
D^{0}(t) \equiv A^{0 *}(t)
$$

in (3.3). Correspondingly, when also $\left(\mathbf{H}_{4}\right)$ holds, in (3.7) we have $D_{1}=D^{0}-A_{1}=A^{0 *}-A_{1}=-A_{2}$, and consequently $D_{2}(t) \equiv 0$ in (3.11), so that in this case (3.10) reduces to the system

$$
\begin{aligned}
-v_{2}^{\prime}(t)+C_{2}(t) u_{2}(t) & =0, \\
u_{2}^{\prime}(t)-v_{2}(t) & =0,
\end{aligned}
$$

and (3.11) becomes

$$
x^{\prime \prime}(t)-C_{2}(t) x(t)=0 .
$$

Finally, if we have the further condition that $C(t)$ is hermitian for 
$t$ on $I$, then one may verify readily that $C^{0}(t), C_{1}(t)$ and $C_{2}(t)$ are also hermitian matrix functions on $I$. Consequently, for self-adjoint systems (2.1) we have the following result.

THEOREM 4.1. Suppose that hypotheses $\left(\mathbf{H}_{1}\right)$ and $\left(\mathbf{H}_{2}\right)$ hold for (2.1), and that $B(t)$ is a continuously differentiable positive definite matrix function on $I$, such that $A(t)$ and $B^{\prime}(t)$ are locally a.c. on $I$. If $M(t)$ is the solution of the corresponding system (3.8), then under the transformation

$$
\begin{aligned}
& u_{2}(t)=M^{*}(t) B^{-1 / 2}(t) u(t), \\
& v_{2}(t)=M^{*}(t)\left[B^{1 / 2}(t) v(t)+A_{1}(t) B^{-1 / 2}(t) u(t)\right],
\end{aligned}
$$

where $A_{1}(t)$ is defined by (3.3), (3.4), the system (2.1) is reduced to the system (4.1), with $C_{2}(t)$ defined by (3.3), (3.7), (3.11).

Under the hypotheses of Theorem 4.1 it may be verified readily that $\left(u_{\alpha}(t) ; v_{\alpha}(t)\right),(\alpha=1,2)$, are conjoined solutions of (2.1) if and only if the associated $\left(u_{2 \alpha}(t) ; v_{2 \alpha}(t)\right)$ defined for (4.3) are conjoined solutions of (4.1). In particular, if $(U(t) ; V(t))$ is a conjoined basis for (2.1) then the associated $\left(U_{2}(t) ; V_{2}(t)\right)$ defined by (4.3) is a conjoined basis for (4.1), and the order of a value $t=t_{0}$ as a focal point of $(U(t) ; V(t))$ for (2.1) is the same as the order of that value as a focal point for the associated conjoined basis $\left(U_{2}(t) ; V_{2}(t)\right)$ for (4.1). Consequently, results on disconjugacy and focal points for one of these differential systems are immediately translatable into results for the associated system. Under the assumption that the hypotheses of Theorem 4.1 hold, let $\Gamma(t ; A, B, C)$ denote the corresponding matrix function $C_{2}(t)$ defined by (3.3), (3.7), (3.11). As examples of such results, we state the following, which are consequences of well-known oscillation and comparison theorems for general self-adjoint systems, (see, for example, Morse [3] and Reid [4, 6, 7]).

THEOREM 4.2. Suppose that the hypotheses of Theorem 4.1 hold, and $[a, b]$ is a compact subinterval of $I$. Then the number of points $t_{0} \in(a, b)$ which are conjugate to $a$, where each conjugate point is counted a number of times equal to its order, is equal to the (negative) index of the hermitian functional

$$
\int_{a}^{b}\left\{\left|\eta^{\prime}(t)\right|^{2}+\eta^{*}(t) \Gamma(t ; A, B, C) \eta(t)\right\} d t
$$

on the class $\mathscr{D}_{0}[a, b]$ of vector functions which are a.c. on $[a, b]$, with $\eta^{\prime}(t) \in \mathbb{R}_{n}^{\infty}[a, b]$, and $\eta(a)=0=\eta(b)$. In particular, (2.1) is disconjugate on $[a, b]$ if and only if the functional (4.4) is positive definite on $\mathscr{D}_{0}[a, b]$. 
THEOREM 4.3. Suppose that $A^{(\alpha)}(t), B^{(\alpha)}(t), C^{(\alpha)}(t), \quad \alpha=1,2$, are triples of matrix functions which individually satisfy the conditions of Theorem 4.2, and for $\alpha=1,2$ let $(2.1)_{\alpha}$ denote the system (2.1) with $(A, B, C)=\left(A^{(\alpha)}, B^{(\alpha)}, C^{(\alpha)}\right)$. If

$$
\Gamma\left(t ; A^{(2)}, B^{(2)}, C^{(2)}\right) \geqq \Gamma\left(t ; A^{(1)}, B^{(1)}, C^{(1)}\right)
$$

for $t \in I$, and $(2.1)_{1}$ is disconjugate on a subinterval $I_{0}$ of $I$, then $(2.1)_{2}$ is also disconjugate on $I_{0}$. Also, if $\left(U_{\alpha}(t) ; V_{\alpha}(t)\right)$ is a conjoined basis for $(2.1)_{\alpha}$, and for $[a, b]$ a compact subinterval of $I$ we have

$$
\left(U_{1}(a) ; V_{1}(a)\right)=\left(U_{2}(a) ; V_{2}(a)\right),
$$

then the number of focal points of $\left(U_{1}(t) ; V_{1}(t)\right)$ on $[a, b]$ is not less than the number of focal points of $\left(U_{2}(t) ; V_{2}(t)\right)$ on $[a, b]$.

It is to be noted that the above reduction of (2.1) to (4.1) is a generalization of a well-known reduction of the scalar differential equation

$$
\left[r(t) u^{\prime}(t)\right]^{\prime}+p(t) u(t)=0
$$

to the canonical form

$$
y^{\prime \prime}(t)+\gamma(t) y(t)=0,
$$

where

$$
\gamma(t)=\left\{\left[r^{\prime}(t)\right]^{2}-2 r(t) r^{\prime \prime}(t)+4 r(t) p(t)\right\} /\left\{4 r^{2}(t)\right\} .
$$

In connection with this particular result, the reader is referred to Birkhoff and Rota [1; p. 38, Exercises 4,6]. In particular, the comparison theorem on the distance between zeros of solutions of a pair of ordinary differential equations which Sather $[8 ; \S 5]$ deduced from properties of solutions of certain boundary problems for hyperbolic partial differential equations may be established readily with the aid of a preliminary translation of the involved intervals of existence, and a subsequent application of the most elementary comparison theorem of the classical Sturmian theory to the associated canonical equations (4.6).

\section{REFERENCES}

1. G. Birkhoff, and G.-C. Rota, Ordinary Differential Equations, Ginn and Company, 1959.

2. P. Hartman, and A. Wintner, On disconjugate differential systems, Canad. J. Math. 8 (1956), 72-81.

3. M. Morse, $A$ generalization of the Sturm separation and comparison theorems in n-space, Math. Ann. 103 (1930), 52-69. 
4. W. T. Reid, Oscillation criteria for linear differential systems with complex coefficients, Pacific J. Math. 6(1956), 733-751.

5. — A Prüfer transformation for differential systems, Pacific J. Math. 8 (1958), 575-584.

6. - Oscillation criteria for self-adjoint differential systems, Trans. Amer. Math. Soc. 101 (1961), 91-106.

7. _ Principal solutions of nonoscillatory linear differential systems, J. Math. Anal. Appl. 9 (1964), 397-423.

8. D. Sather, Maximum and monotoneity properties of initial-boundary value problems for hyperbolic equations, Pacific J. Math. 19 (1966), 141-157.

Received March 31, 1970. This research was supported by the Air Force Office of Scientific Research', Office of Aerospace Research, United States Air Force, under Grant AF-AFOSR-68-1398.

The University of OKLahoma

NORMAN, OKLAHOMA 


\section{PACIFIC JOURNAL OF MATHEMATICS}

\section{EDITORS}

\author{
H. SAMELSON \\ Stanford University \\ Stanford, California 94305 \\ Richard Pierce \\ University of Washington \\ Seattle, Washington 98105
}

\author{
J. DugundJI \\ Department of Mathematics \\ University of Southern California \\ Los Angeles, California 90007 \\ RICHARD ARENS \\ University of California \\ Los Angeles, California 9.0024
}

\section{ASSOCIATE EDITORS}

\begin{tabular}{|c|c|}
\hline E. F. BECKENBACH & K. YoshidA \\
\hline \multicolumn{2}{|c|}{ SUPPORTING INSTITUTIONS } \\
\hline UNIVERSITY OF BRITISH COLUMBIA & STANFORD UNIVERSITY \\
\hline CALIFORNIA INSTITUTE OF TECHNOLOGY & UNIVERSITY OF TOKYO \\
\hline UNIVERSITY OF CALIFORNIA & UNIVERSITY OF UTAH \\
\hline MONTANA STATE UNIVERSITY & WASHINGTON STATE UNIVERSITY \\
\hline UNIVERSITY OF NEVADA & UNIVERSITY OF WASHINGTON \\
\hline NEW MEXICO STATE UNIVERSITY & * \\
\hline OREGON STATE UNIVERSITY & AMERICAN MATHEMATICAL SOCIETY \\
\hline UNIVERSITY OF OREGON & CHEVRON RESEARCH CORPORATION \\
\hline OSAKA UNIVERSITY & TRW SYSTEMS \\
\hline UNIVERSITY OF SOUTHERN CALIFORNIA & NAVAL WEAPONS CENTER \\
\hline
\end{tabular}

The Supporting Institutions listed above contribute to the cost of publication of this Journal, but they are not owners or publishers and have no responsibility for its content or policies.

Mathematical papers intended for publication in the Pacific Journal of Mathematics should be in typed form or offset-reproduced, (not dittoed), double spaced with large margins. Underline Greek letters in red, German in green, and script in blue. The first paragraph or two must be capable of being used separately as a synopsis of the entire paper. The editorial "we" must not be used in the synopsis, and items of the bibliography should not be cited there unless absolutely necessary, in which case they must be identified by author and Journal, rather than by item number. Manuscripts, in duplicate if possible, may be sent to any one of the four editors. Please classify according to the scheme of Math. Rev. Index to Vol. 39. All other communications to the editors should be addressed to the managing editor, Richard Arens, University of California, Los Angeles, California, 90024.

50 reprints are provided free for each article; additional copies may be obtained at cost in multiples of 50 .

The Pacific Journal of Mathematics is published monthly. Effective with Volume 16 the price per volume (3 numbers) is $\$ 8.00$; single issues, $\$ 3.00$. Special price for current issues to individual faculty members of supporting institutions and to individual members of the American Mathematical Society: $\$ 4.00$ per volume; single issues $\$ 1.50$. Back numbers are available.

Subscriptions, orders for back numbers, and changes of address should be sent to Pacific Journal of Mathematics, 103 Highland Boulevard, Berkeley, California, 94708.

PUBLISHED BY PACIFIC JOURNAL OF MATHEMATICS, A NON-PROFIT CORPORATION

Printed at Kokusai Bunken Insatsusha (Internatıonal Academic Printing Co., Ltd.), 7-17, Fujimi 2-chome, Chiyoda-ku, Tokyo, Japan. 


\section{Pacific Journal of Mathematics}

\section{Vol. 35, No. $3 \quad$ November, 1970}

John D. Arrison and Michael Rich, On nearly commutative degree one algebras . . . 533

Bruce Alan Barnes, Algebras with minimal left ideals which are Hilbert spaces . . . . 537

Robert F. Brown, An elementary proof of the uniqueness of the fixed point index . . . 549

Ronn L. Carpenter, Principal ideals in F-algebras .................... 559

Chen Chung Chang and Yiannis (John) Nicolas Moschovakis, The Suslin-Kleene

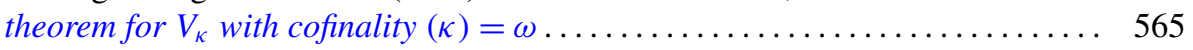

Theodore Seio Chihara, The derived set of the spectrum of a distribution

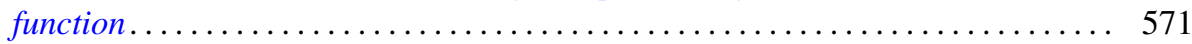

Tae Geun Cho, On the Choquet boundary for a nonclosed subspace of $C(S) \ldots \ldots \quad 575$

Richard Brian Darst, The Lebesgue decomposition, Radon-Nikodym derivative,

conditional expectation, and martingale convergence for lattices of sets .......

David E. Fields, Dimension theory in power series rings . . . . . . . . . . . .

Michael Lawrence Fredman, Congruence formulas obtained by counting

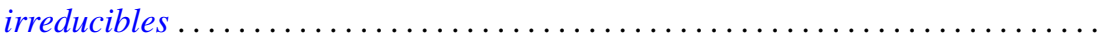

John Eric Gilbert, On the ideal structure of some algebras of analytic functions.....

G. Goss and Giovanni Viglino, Some topological properties weaker than

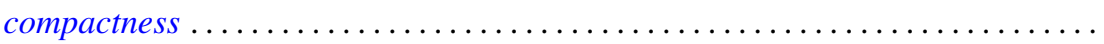

581

601

625

George Grätzer and J. Sichler, On the endomorphism semigroup (and category) of

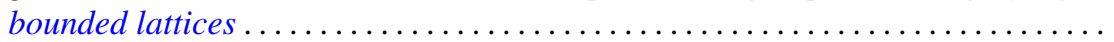

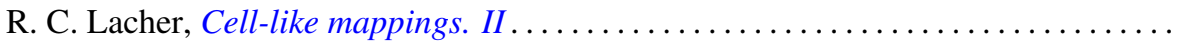

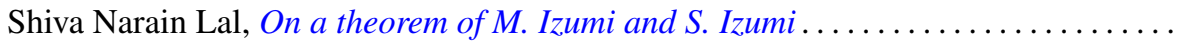

661

Howard Barrow Lambert, Differential mappings on a vector space ...............

Richard G. Levin and Takayuki Tamura, Notes on commutative power joined

semigroups.

Robert Edward Lewand and Kevin Mor McCrimmon, Macdonald's theorem for quadratic Jordan algebras.

J. A. Marti, On some types of completeness in topological vector spaces ....

Walter J. Meyer, Characterization of the Steiner point

717

Saad H. Mohamed, Rings whose homomorphic images are $q$-rings ...

727

Thomas V. O'Brien and William Lawrence Reddy, Each compact orientable surface

of positive genus admits an expansive homeomorphism ...

737

Robert James Plemmons and M. T. West, On the semigroup of binary relations...

743

Calvin R. Putnam, Unbounded inverses of hyponormal operator . .

755

William T. Reid, Some remarks on special disconjugacy criteria for differential

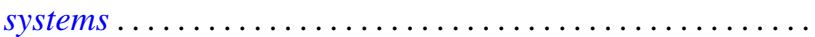

C. Ambrose Rogers, The convex generation of convex Borel sets in euclidean

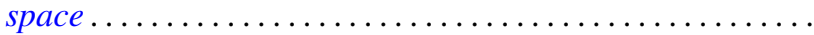

S. Saran, A general theorem for bilinear generating functions .

S. W. Smith, Cone relationships of biorthogonal systems ......

Wolmer Vasconcelos, On commutative endomorphism rings ....

795

Vernon Emil Zander, Products of finitely additive set functions from Orlicz

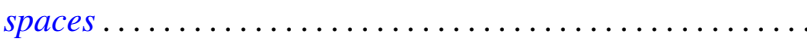

G. Sankaranarayanan and C. Suyambulingom, Correction to: "Some renewal

theorems concerning a sequence of correlated random variables" .

Joseph Zaks, Correction to: "Trivially extending decompositions of $E^{n}$ ”....... 805

Dong Hoon Lee, Correction to: "The adjoint group of Lie groups" ............ 805

James Edward Ward, Correction to: "Two-groups and Jordan algebras". 